\title{
Gapless unidirectional photonic transport using all-dielectric kagome lattices
}

\author{
Stephan Wong, ${ }^{1,2, *}$ Matthias Saba, ${ }^{2,3}$ Ortwin Hess, ${ }^{2,4}$ and Sang Soon Oh $\oplus^{1, \dagger}$ \\ ${ }^{1}$ School of Physics and Astronomy, Cardiff University, Cardiff CF24 3AA, United Kingdom \\ ${ }^{2}$ The Blackett Laboratory, Imperial College London, London SW7 2AZ, United Kingdom \\ ${ }^{3}$ Adolphe Merkle Institute, University of Fribourg, 1700 Fribourg, Switzerland \\ ${ }^{4}$ School of Physics, CRANN \& AMBER, Trinity College Dublin, Dublin 2, Ireland
}

(Received 19 July 2019; revised manuscript received 3 December 2019; published 13 January 2020)

\begin{abstract}
Photonic topological insulators are a promising photonic platform due to the possibility of unidirectional edge states with insensitivity to bending, fabrication imperfections, or environmental fluctuation. Here we demonstrate highly efficient unidirectional photonic edge mode propagation facilitated by an optical analog of the quantum valley Hall effect. With an all-dielectric kagome lattice design, we demonstrate broadband suppressed reflection in the presence of sharp corners and further show negligible vertical losses in a semiconductor-based device at telecommunication wavelengths.
\end{abstract}

DOI: 10.1103/PhysRevResearch.2.012011

When propagating in a (structured) material or waveguide, not all of the light travels in this initial direction but parts of it experience such back-reflection due to bending, fabrication defects, or environmental variations. For most applications, back-propagation should be avoided and it is thus not surprising that the unique properties of photonic topological insulators (PTIs) [1,2] have attracted widespread attention due to their promise to prohibit back-reflections. The basis of such back-scattering-free one-way waveguides lies at the interface of two topologically inequivalent photonic crystals (PhCs) which exhibit topological edge modes that - guaranteed by the bulk-boundary correspondence [3]propagate only in one direction and are at the same time robust against perturbations. Not surprisingly, a plethora of possible topologically nontrivial photonic designs has been put forward, involving nonreciprocal systems [4], complex metamaterials [5], the Floquet topological insulator principle [6], and an artificial magnetic gauge [7,8]. However, the aforementioned PTIs need strong magnetic fields, are complicated to fabricate, and/or are difficult if not impossible to scale to optical frequencies.

As an alternative, a deformed honeycomb-based topological $\mathrm{PhC}$ [9], which emulates the quantum spin Hall effect (QSHE) [9-12] has recently gained interest, not least due to its simple fabrication as compared to other PTIs. Nevertheless, while 2D hexagonal symmetries (such as the honeycombbased topological $\mathrm{PhC}$ ) generally lead to Dirac cones at the $K$ and $K^{\prime}$ points of the Brillouin zone (BZ), and with a geometrical perturbation it is possible to lift the pointlike

\footnotetext{
*wongs16@ cardiff.ac.uk

†ohs2@cardiff.ac.uk
}

Published by the American Physical Society under the terms of the Creative Commons Attribution 4.0 International license. Further distribution of this work must maintain attribution to the author(s) and the published article's title, journal citation, and DOI. degeneracies in order to obtain a nontrivial topological and complete photonic band gap [13] (which leads to topological protection defined within the parameter space of a certain type of a deterministic geometrical perturbation that differs from the traditional Hatsugai sense [3]), there is an inherent problem. The pseudo-time-reversal antiunitary operator $\mathcal{T}^{2}=-\mathbb{1}$, introduced to have well-defined orthogonal spin up/down channels, is constructed on the basis of the sixfold rotation $\left(C_{6}\right)$ operator of the crystal. However, the $C_{6}$ symmetry of the crystal is broken in any finite, truncated, configuration and the spin up and spin down channels couple to each other. Consequently, while edge modes are guaranteed at the interface between the two topologically distinct deformed honeycomb PhCs, for most frequencies within the band gap, there is an anticrossing in their dispersion and they eventually do suffer from intrinsic back-reflection. Yet, starting from a $C_{6 v}$ symmetry with symmetry protected Dirac cones, it can be shown that there are two routes towards breaking the symmetry to open a topological band gap without breaking optical reciprocity [13]: one related to the aforementioned QSHE [10], and the other to the quantum valley Hall effect (QVHE) [14]. The QVHE has been widely studied in photonic and mechanical systems with staggered honeycomb [15-21] lattice, triangular rods [22-24], or multipod [25-27] structure.

Here, we introduce an all-dielectric PTI based on a kagome lattice [28] that naturally lends itself to QVHE symmetry breaking [13], while being composed of monodisperse rods of a single dielectric material. Compared to the QVHE designs of triangular-like holes/rods array and staggered honeycomb structures, the proposed perturbed kagome lattice requires a single monodisperse type of circular holes/rods and is thus easier fabricated. It is this simplicity of the kagome-based design in terms of fabrication and its unidirectional edge mode transport which makes it an ideal candidate for practical applications at near-infrared and visible wavelengths. Here, we present and model the predicted behavior for an on-chip platform that can be readily fabricated with state-of-the-art semiconductor growth techniques [29]. 
(a)

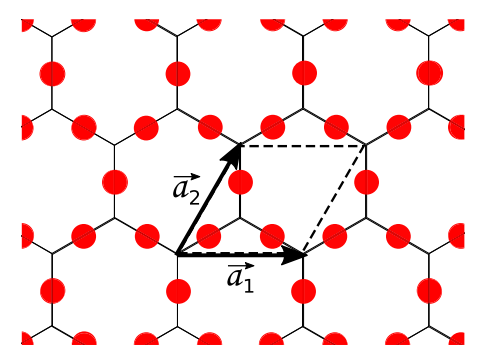

(b)
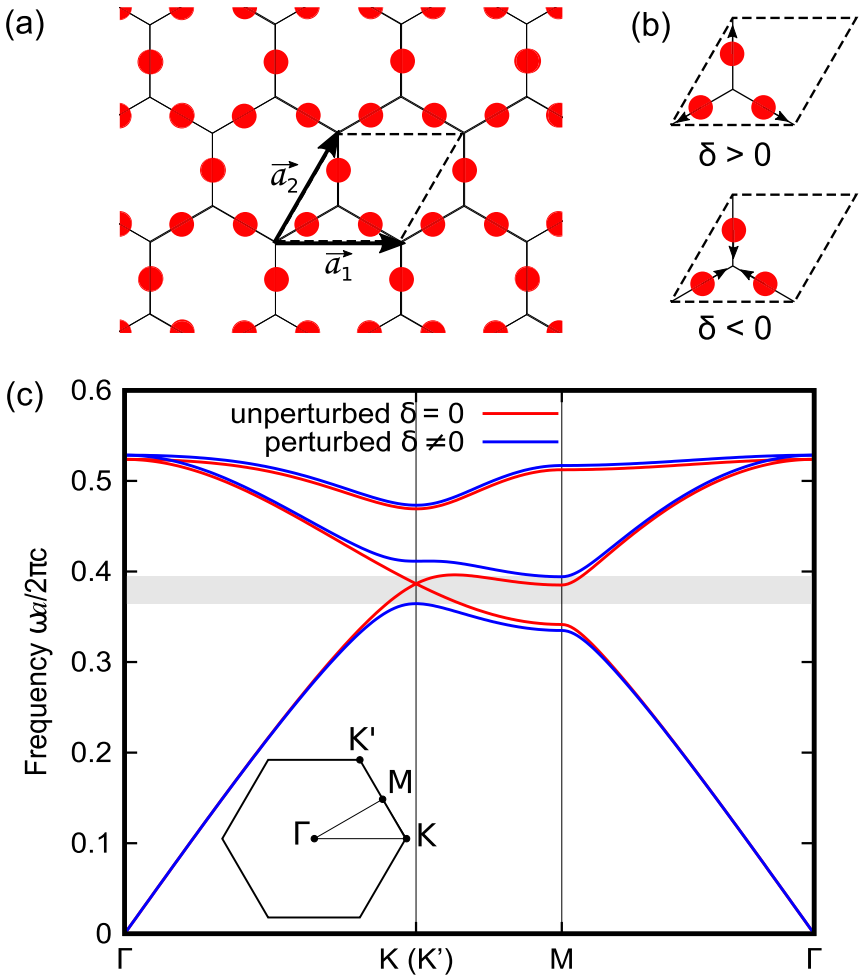

FIG. 1. (a) Schematic of a kagome lattice. The solid line is a guide to the eye for the hexagonal symmetry. (b) Perturbations are considered by putting the rods further away (top) or closer (down) to each other, represented by the arrows. (c) Band structure of the kagome lattice for the unperturbed (solid red line) and perturbed (solid blue line) cases. The inset shows the first Brillouin zone.

The kagome lattice, named after a traditional Japanese basketweave pattern [28], has lattice sites at the midpoints of the edges in the regular hexagonal wallpaper tiling $\{6,3\}$, as illustrated in Fig. 1(a). The unit cell is here composed of three rods and the perturbation to lift the degeneracy can be introduced such that these rods get closer (negative perturbation $\delta<0$ ) or further away (positive perturbation $\delta>0$ ) from their shared corner of the hexagons [Fig. 1(b)]: $\boldsymbol{r} \mapsto \boldsymbol{r}^{\prime}=(1 \pm \delta) \boldsymbol{r}$, where $\boldsymbol{r}$ is a vector taken from the corner of a hexagon to the adjacent rod.

Importantly, the perturbation opens a band gap by lifting the linear degeneracy at the $K$ and $K^{\prime}$ points which is symmetry-induced in the unperturbed case. This generic behavior manifests itself in the photonic band structure for $E_{z}$ out-of-plane transverse magnetic (TM) polarization [Fig. 1(c)] obtained with the open-source software MIT Photonic Bands (MPB) [30] for the unperturbed $\delta=0$ and perturbed $\delta= \pm 0.15$ case. The model kagome photonic crystal, with lattice constant $a$, is composed of rods with diameter $d=0.2 a$ and permittivity $\varepsilon=12$ within a vacuum $(\varepsilon=1)$ background. In contrast to the perturbed honeycomb $\mathrm{PhC}$ [9], the translation symmetry is conserved after the perturbation, i.e., the primitive lattice vectors (albeit not unique) are themselves preserved. As a consequence, the reciprocal lattice vectors remain the same and therefore the $K$ and $K^{\prime}$ points remain distinct and do not map to the $\Gamma$ point as is the case for the perturbed honeycomb lattice [9].
To describe the band gap opening more rigorously, we adopt a perturbation-based group theoretical approach detailed in Refs. $[13,31]$ to the hexagonal wallpaper group p $6 \mathrm{~mm}$ (17). Then, an effective Hamiltonian is derived for a (small) geometrical perturbation, $\tilde{\delta}$, and close to $\mathrm{K}, \delta \boldsymbol{k}=\left(\delta k_{x}, \delta k_{y}\right)$, in the canonical basis of the induced $\mathrm{K}$ irreducible representation (irrep) from the 2D little group irrep of $p 6 \mathrm{~mm}$ [32]:

$$
\mathcal{H}_{\text {kagome }}=\delta k_{x} \gamma_{1}-\delta k_{y} \gamma_{2}+\tilde{\delta} \gamma_{5}=\left(\begin{array}{cc}
\mathcal{W}_{K} & 0 \\
0 & \mathcal{W}_{K^{\prime}}
\end{array}\right),
$$

where $\gamma_{1}:=\sigma_{3} \otimes \sigma_{3}, \gamma_{2}:=\sigma_{3} \otimes \sigma_{1}, \gamma_{3}:=\sigma_{1} \otimes \mathbb{1}_{2}, \gamma_{4}:=\sigma_{2} \otimes \mathbb{1}_{2}$, and $\gamma_{5}:=\sigma_{3} \otimes \sigma_{2}$ are matrices satisfying a Clifford algebra generated by the vector field spanned by the $\gamma_{i}$, with the associated anticommutation relation $\left\{\gamma_{i}, \gamma_{j}\right\}=2 \delta_{i j} \mathbb{1}_{4} . \mathcal{W}_{K / K^{\prime}}= \pm$ $\boldsymbol{h} \cdot \boldsymbol{\sigma}$ represent Weyl Hamiltonians of opposite chirality in the vicinity of $K / K^{\prime}$ with $\boldsymbol{h}=\left(-\delta k_{y}, \tilde{\delta}, \delta k_{x}\right)$ and $\boldsymbol{\sigma}=\left(\sigma_{1}, \sigma_{2}, \sigma_{3}\right)$ the Pauli matrix vector. In Eq. (1), $\tilde{\delta}$ models the geometrical perturbation and therefore is proportional to the displacement of the rods $\delta$ away from the one of the unperturbed lattice. The eigenvalues $E$ of the Weyl Hamiltonian at the $K / K^{\prime}$ point are $E= \pm \sqrt{\delta k_{x}^{2}+\delta k_{y}^{2}+\tilde{\delta}^{2}}$ confirming that we obtain a linear degeneracy (Dirac point) in the 2D BZ for $\tilde{\delta}=0$, i.e., for the unperturbed kagome lattice, and have a band gap for $\tilde{\delta} \neq 0$.

Additionally, from Eq. (1) it is clear that the effective Hamiltonian $\mathcal{H}_{\text {kagome }}$ is necessarily block-diagonalized [32] in its canonical basis as in the case with the QSHE Kane-Mele Hamiltonian [10], because translation symmetry is not broken with the perturbation. It does not mix the $K / K^{\prime}$ irrep of the invariant translation group. The $K / K^{\prime}$ points thus play the role of two orthogonal pseudo-spin channels, known as the valley degree of freedom, with the generating unitary pseudo-timereversal operator $\tilde{\mathcal{T}}=\sigma_{3} \otimes \mathbb{1}_{2}$ with $\left[\tilde{\mathcal{T}}, \mathcal{H}_{\text {kagome }}\right]=0$, invariant under rotational symmetry breaking.

To show how the proposed kagome design emulates the QVHE emerging from the nontrivial Dirac points in the 2D $\mathrm{BZ}$, we use the valley Chern number defined at the $K$ and $K^{\prime}$ points [15-20,22-27]:

$$
C_{K / K^{\prime}}=\frac{1}{2 \pi} \int_{S_{K / K^{\prime}}} \mathcal{F}(\boldsymbol{k}) d^{2} \boldsymbol{k} .
$$

The integration is performed over the two valley domains $S_{K / K^{\prime}}$ defined in Fig. 2 as the two triangles which together form the dual of the standard hexagonal BZ tessellation of reciprocal space. The integrand is the Berry curvature [33] $\mathcal{F}(\boldsymbol{k})=\nabla \times i\left\langle\boldsymbol{u}_{\boldsymbol{k}} \mid \nabla_{\boldsymbol{k}} \boldsymbol{u}_{\boldsymbol{k}}\right\rangle$. Note that $C_{K / K^{\prime}}$ is not a quantized topological invariant because $S_{K / K^{\prime}}$ is not a closed surface, so that the Chern theorem does not hold. Figure 2 shows the Berry curvatures calculated for a positively $(\delta=+0.15)$ and negatively $(\delta=-0.15)$ deformed kagome lattice, for $\boldsymbol{k}$ points in the reciprocal primitive unit cell. They are directly calculated from the numerical field profile using a Wilson loop approach to numerically avoid random gauge dependence (see Ref. [34] for the details of the calculation). The valley Chern number obtained for positive (negative) perturbation by integrating around the $K / K^{\prime}$ are then $C_{K / K^{\prime}}= \pm 0.18$ $\left(C_{K / K^{\prime}}=\mp 0.18\right)$. It can be shown that the valley Chern number depends on the perturbation strength, and explains why we can only get a valley Chern number $C_{K / K^{\prime}}= \pm 1 / 2$ for 

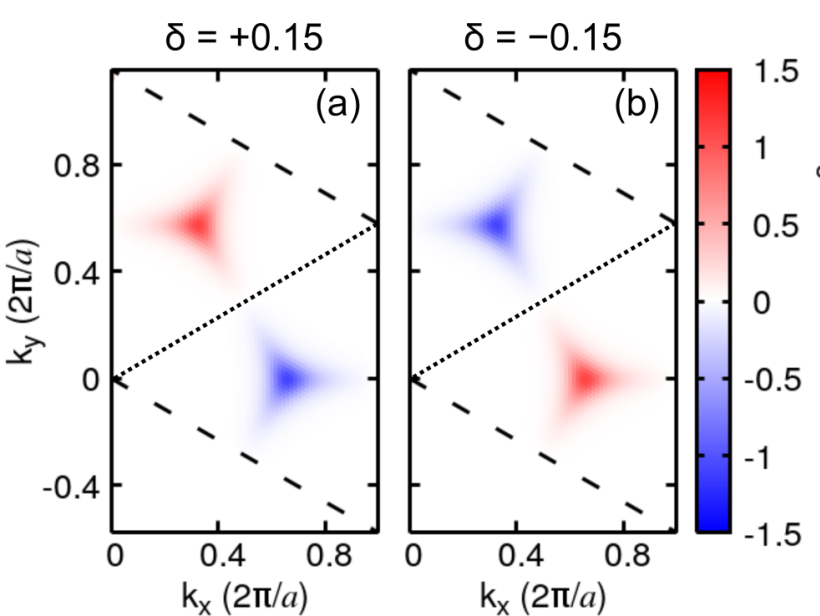

FIG. 2. Calculated Berry curvatures in the reciprocal primitive unit cell, using a Wilson loop approach for (a) positively and (b) negatively perturbed kagome lattices. The dashed line represents the limit of the reciprocal primitive unit cell and the dotted black line defines the boundary for the regions of integration $S_{K / K^{\prime}}$ in Eq. (2).

infinitesimal perturbation, as reported before [15-20,22-27] (see Ref. [34] for more details on the valley Chern number).

Although the valley Chern number is not a topological invariant, a strong bulk-boundary correspondence similar to Ref. [3] exists in the extended parameter space $\left(-\delta k_{y}, \tilde{\delta}, \delta k_{x}\right)$ where Weyl charges of opposite chirality lead to guaranteed edge modes in the $K / K^{\prime}$ valleys, respectively [13]. These Weyl charges can be correlated one-to-one to the sign of the valley Chern numbers, which can thus be interpreted as a topological integer with associated bulk-boundary correspondence. In contrast to Ref. [3], however, the existence of the strong correspondence in the extended parameter space only fixes crystal termination and is only valid for inclinations for which the $K$ and $K^{\prime}$ point are not projected to the same point in the edge BZ (cf. BZ insets in Fig. 3). The bulk-boundary correspondence thus reduces to the weaker form which rigorously valid only for specific well-defined boundaries. From the expression of the effective Hamiltonian $\mathcal{H}_{\text {kagome }}$, it is evident that a nontrivial Weyl charge is located at the $K$ and $K^{\prime}$ points which has opposite signs because $\mathcal{W}_{K}=-\mathcal{W}_{K^{\prime}}$ and which has opposite signs for opposite perturbation strength. As a consequence, and from Fig. 2, starting with an unperturbed lattice and then perturbing positively on one side of a chosen interface and negatively on the other could potentially lead to topological edge modes at most frequencies.

Figure 3 shows the corresponding supercell band structure for $\delta= \pm 0.15$ and for different interface inclinations. The solid blue lines correspond to the bulk modes and the solid colored lines correspond to topological edge modes inside the bulk band gap. This figure shows that an anticrossing arises when the $K / K^{\prime}$ points are projected onto the same $k_{\|}$ points $[\Gamma-M$ inclination, Fig. 3(b)] while a crossing arises for $\Gamma-K$ inclination [Fig. 3(a)]. In the $\Gamma-K$ inclination case [Fig. 3(a)], each interface supports two counter-propagating edge modes corresponding to the well-defined pseudospin up (down) edge modes of the $\mathrm{K}\left(K^{\prime}\right)$ valleys. These result from the opposite Weyl charges at the $K$ (or $K^{\prime}$ ) point for two sides of the interface $[3,13]$. In the $\Gamma-M$ inclination case
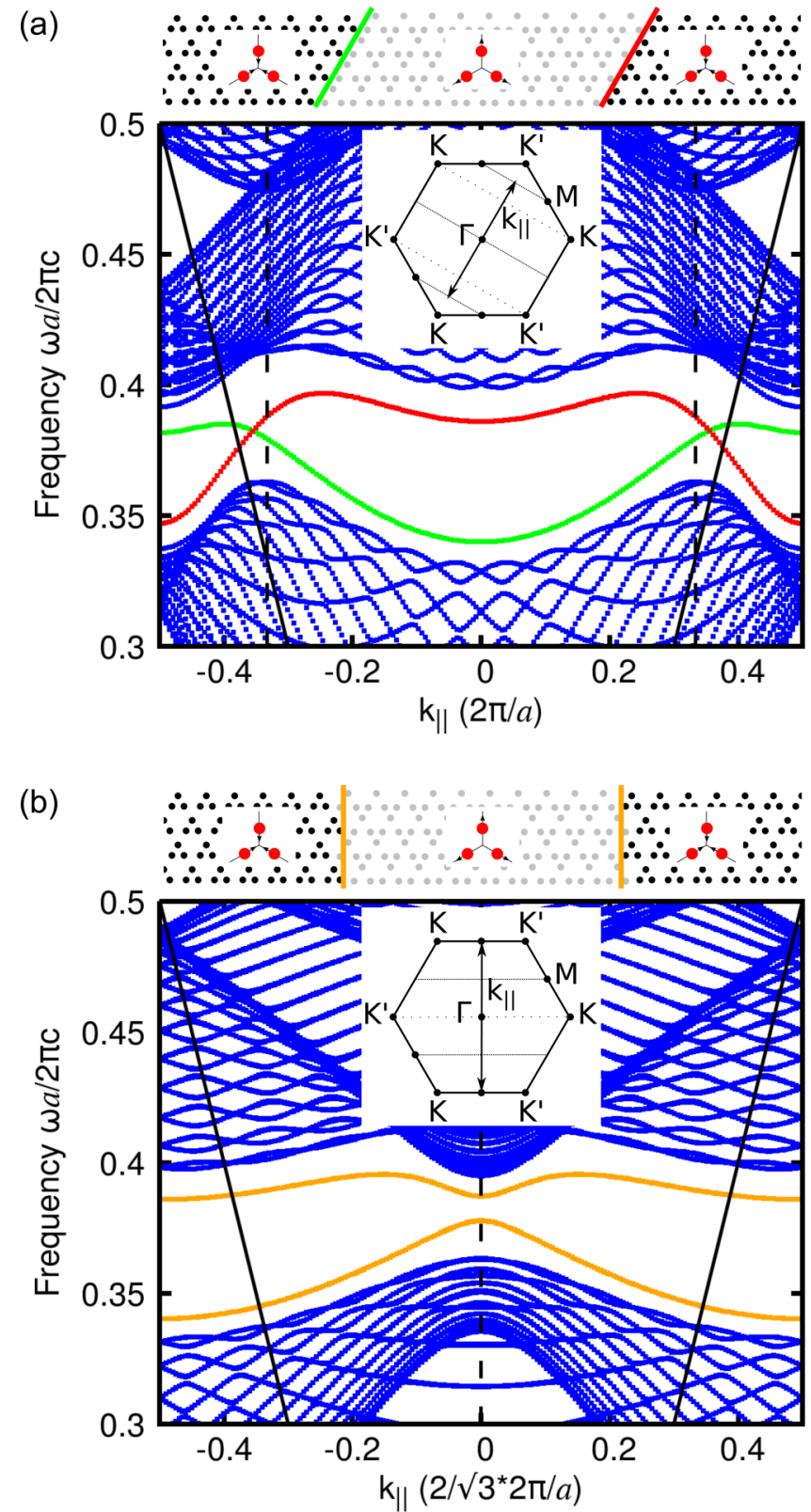

FIG. 3. Supercell band structure at the interface between two topologically distinct kagome photonic crystals along different inclination directions: (a) $\Gamma-K$ and (b) $\Gamma-M$. The front and back interfaces are represented by solid lines with different colors on both the sketch and the band structure in (a). In (b), the interfaces and the edge mode dispersions are represented by the same color because the two interfaces are equivalent. The insets show the $k_{\|}$sweep direction. The solid black line represents the light lines. The vertical dashed lines mark the position of $K / K^{\prime}$. The parameters are the same as in Fig. 1 and with perturbation strength $\delta= \pm 0.15$.

[Fig. 3(b)], however, the pseudospin separation breaks down and the edge modes suffer from back-scattering, similarly to the honeycomb $\mathrm{PhC}$ [9].

We here demonstrate broadband back-scattering-immunity numerically in Fig. 4 for finite perturbations, going beyond what has been shown rigorously for infinitesimal perturbation strengths with our generic theory based on symmetry only. The unidirectional propagation with negligible intervalley 

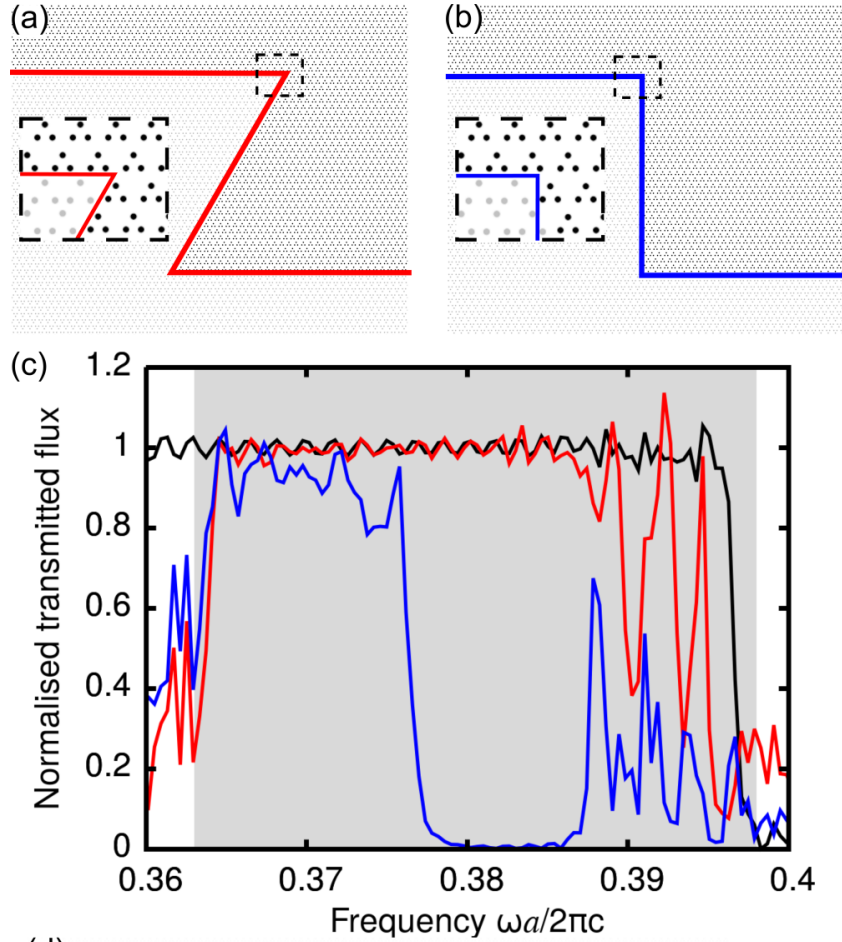

(d)

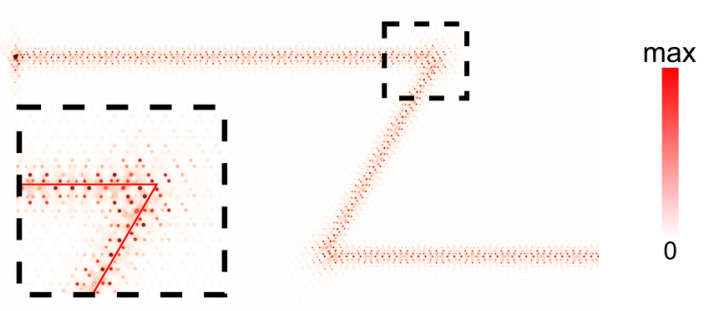

FIG. 4. [(a) and (b)] Waveguides where the bending has a (a) $Z$ shape or (b) staircase shape where the bright (dark) rods are the positively (negatively) perturbed kagome lattice. Insets are zoomed in of the interface at the dashed square region. (c) Transmission spectra for a waveguide without any bending (solid black line), $Z$ shaped (solid red line), or staircase-shaped (solid blue line) bending. The shaded area is a guide for the band gap frequency range. The parameters are kept the same as in Fig. 3. (d) Power of the edge modes excited at the $Z$-shaped bending structure.

coupling is demonstrated by studying the transmission through waveguides with bendings of different inclinations. Figures 4(a) and 4(b) shows two examples of waveguides oriented in a $\Gamma-K$ inclination direction in which a bending is introduced such that the projected wave vectors of $K$ and $K^{\prime}$ onto the $k_{\|}$line are distinct in a $Z$-shaped design [Fig. 4(a), inset Fig. 3(a)], or fall onto the same point along the vertical interface in a staircase-shaped design [Fig. 4(b), inset Fig. 3(b)]. In the case of the $Z$-shaped waveguide, we focus on the red interface highlighted in Fig. 3(a) (which is not equivalent to the green interface). Figure 4(c) shows the normalized transmitted flux at the end of the waveguides, obtained using MIT electromagnetic equation propagation (MEEP) [35]. At high frequencies, around the upper edge of the photonic bulk band gap, large oscillations are observed due to the presence of additional modes that couple with the two valley channels (see Fig. 3 and Ref. [34] for more information on the modal

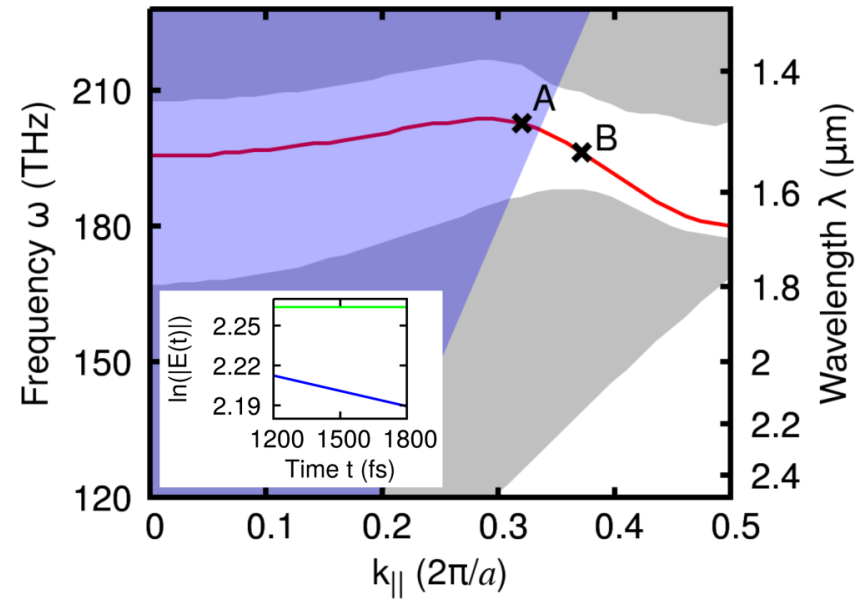

FIG. 5. Calculated band structure of the PhC slab along the front, red, interface configuration [see Fig. 3(a)]. The grey region is the projected bulk bandstructure, the red line is the edge mode dispersion and the purple shaded area represents the light cone. Modes at $A$ and $B$ are above and below the light line, respectively. The inset shows the temporal decay of the mode at point $A$ (blue curve) and $B$ (green curve).

transmissions). The transmission for the $Z$-shaped waveguide (solid red line) is of the same order of magnitude as for a straight waveguide with no bending (solid black line) for most of the frequencies inside the band gap (represented by the grey shaded region). In contrast, the staircase-shaped bending (solid blue line) leads to substantial back-reflection resulting in lower transmission because of the non-negligible inter-valley couplings on the $\Gamma-M$ inclination interface. This $\Gamma-M$ inclination bending additionally introduces a band gap in the dispersion where the edge mode cannot propagate. Broadband robust transmission is therefore achieved for the $\Gamma-K$ inclination and in practice the orthogonality of two pseudo-spin channels at the wave vectors away from $K / K^{\prime}$ is as good as at $K / K^{\prime}$. Figure $4(\mathrm{~d})$ shows the power profile for a mode propagating along the interface with bendings at frequency $\omega=0.37(2 \pi c / a)$ with negligible back-reflection. Here, the unidirectional edge mode has been excited using a rotating magnetic point dipole source $[9,36]$ and the spatial position of the source has been determined using a chirality map [37] (see Ref. [34] for more details on the chirality map).

From a practical point of view, for the case of a $\Gamma-K$ interface inclination, edge modes lie close to or below the light line, thus improving vertical mode confinement without the need of sandwiching the waveguides with mirrors [9]. To make use of the better confinement, we propose a design composed of an InGaAsP free-standing $\mathrm{PhC}$ slab ( $n=3.3$ [29]) of 170-nm thickness. Fabrication of this structure can be carried out using a III-V semiconductor wafer consisting of an InGaAsP substrate. The pattern of the air holes can be defined by standard electron-beam lithography and inductively coupled plasma reactive-ion etching. Importantly, the proposed structure can be designed to work at telecommunication wavelengths, from 1.2 to $1.8 \mu \mathrm{m}$, as illustrated in Fig. 5. The supercell band structure for TE-like modes (even-symmetric electric fields) is calculated with a finite-difference time-domain (FDTD) solver [38] for a lattice 
(a)

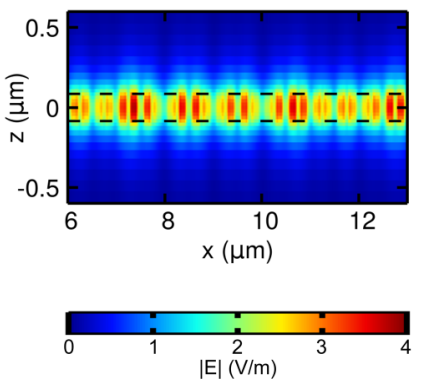

FIG. 6. Field profile of the edge modes lying below the light line (point $B$ in Fig. 5) in (a) the $y=0$ plane and (b) the $z=0$ plane. The dashed lines are a guide to the eye for the $z$ limit of the free-standing slab.

constant $a=0.5 \mu \mathrm{m}$, air-holes with diameter $d=0.3 a=150$ $\mathrm{nm}$ and perturbation $\delta= \pm 0.15$. The band gap ranges from 1.48 to $1.58 \mu \mathrm{m}$ corresponding to a mid-gap at $1.53 \mu \mathrm{m}$. Since the materials are assumed to be lossless in our simulations, any temporal decay, quantified by $\gamma$ in $E(t) \propto e^{i(\omega+i \gamma) t}$, is due to the leakiness of the mode. The inset of Fig. 5 shows the slope of the curves corresponding to $\gamma$, and demonstrating a negligible $\gamma_{B}$ for the mode below the light line (point $B$ ), whereas a finite leakage $\gamma_{A}$ is present for the mode lying above the light line (point $A$ ). Figures 6(a) and 6(b) shows the electric field distribution corresponding to the point $B$ in Fig. 5. Out-of-plane confinement is guaranteed by the mode's presence below the light line.

[1] L. Lu, J. D. Joannopoulos, and M. Soljačić, Nat. Phys. 12, 626 (2016).

[2] T. Ozawa, H. M. Price, A. Amo, N. Goldman, M. Hafezi, L. Lu, M. C. Rechtsman, D. Schuster, J. Simon, O. Zilberberg, and I. Carusotto, Rev. Mod. Phys. 91, 015006 (2019).

[3] Y. Hatsugai, Phys. Rev. Lett. 71, 3697 (1993).

[4] F. D. M. Haldane and S. Raghu, Phys. Rev. Lett. 100, 013904 (2008).

[5] A. B. Khanikaev, S. Hossein Mousavi, W.-K. Tse, M. Kargarian, A. H. MacDonald, and G. Shvets, Nat. Mater. 12, 233 (2013).

[6] M. C. Rechtsman, J. M. Zeuner, Y. Plotnik, Y. Lumer, D. Podolsky, F. Dreisow, S. Nolte, M. Segev, and A. Szameit, Nature (London) 496, 196 (2013).

[7] M. Hafezi, E. A. Demler, M. D. Lukin, and J. M. Taylor, Nat. Phys. 7, 907 (2011).

[8] K. Fang, Z. Yu, and S. Fan, Nat. Photon. 6, 782 (2012).

[9] L.-H. Wu and X. Hu, Phys. Rev. Lett. 114, 223901 (2015).

[10] C. L. Kane and E. J. Mele, Phys. Rev. Lett. 95, 146802 (2005).

[11] H. Chen, H. Nassar, A. N. Norris, G. K. Hu, and G. L. Huang, Phys. Rev. B 98, 094302 (2018).

[12] Y. Yang, Y. F. Xu, T. Xu, H.-X. Wang, J.-H. Jiang, X. Hu, and Z. H. Hang, Phys. Rev. Lett. 120, 217401 (2018).

[13] M. Saba, S. Wong, M. Elman, S. S. Oh, and O. Hess, arXiv:1911.12132.

[14] Y. Kim, K. Choi, J. Ihm, and H. Jin, Phys. Rev. B 89, 085429 (2014).

In summary, we have introduced a new kagome-like photonic topological insulator emulating the optical analog of the quantum valley Hall effect. We have numerically shown that the associated topological edge modes do not intrinsically suffer from back-scattering for tailored inclinations. For $\Gamma-K$ inclination, the coupling between pseudospin channels is shown to be negligible while edge modes are guaranteed in the center of the band gap. Based on our theoretical predictions, we have presented a realistic $3 \mathrm{D}$ design that can be fabricated with state-of-the-art methods [29], and works at suitable wavelengths for telecommunication applications. We have demonstrated improved vertical confinement due to edge modes lying below the light line. The simplicity of the proposed design structure due to its monodisperse rods/holes makes it possible to fabricate it using many conventional techniques such as selective-area epitaxy or electron-beam patterning. We have also shown that possible challenges resulting from low filling ratio can be overcome by tuning the perturbation making the band gap of about $100 \mathrm{~nm}$ at telecommunication wavelengths. Work has been done on kagome lattice in photonic systems [39]. Complementing previous work with kagome lattices, we have provided an in-depth analysis by explaining the reason of possible back-reflection translated in the transmission spectra and analyzing the out-of-plane loss in 3D realistic design.

This work is part funded by the European Regional Development Fund through the Welsh Government [80762-CU145 (East)]. We would like to thank Anthony J. Bennett, Yongkang Gong, and Andreas Pusch for helpful discussions.

[15] X. D. Chen, F. L. Zhao, M. Chen, and J. W. Dong, Phys. Rev. B 96, 020202(R) (2017).

[16] J. Dong, X. D. Chen, H. Zhu, Y. Wang, and X. Zhang, Nat. Mater. 16, 298 (2017).

[17] O. Bleu, D. D. Solnyshkov, and G. Malpuech, Phys. Rev. B 95, 235431 (2017).

[18] X. D. Chen, F. L. Shi, H. Liu, J. C. Lu, W. M. Deng, J. Y. Dai, Q. Cheng, and J. W. Dong, Phys. Rev. Appl. 10, 044002 (2018).

[19] Z. Gao, Z. Yang, F. Gao, H. Xue, Y. Yang, J. Dong, and B. Zhang, Phys. Rev. B 96, 201402(R) (2017).

[20] X. T. He, E. T. Liang, J. J. Yuan, H. Y. Qiu, X. D. Chen, F. L. Zhao, and J. W. Dong, Nat. Commun. 10, 872 (2019).

[21] R. K. Pal and M. Ruzzene, New J. Phys. 19, 025001 (2017).

[22] T. Ma and G. Shvets, New J. Phys. 18, 025012 (2016).

[23] M. I. Shalaev, W. Walasik, A. Tsukernik, Y. Xu, and N. M. Litchinitser, Nat. Nanotechnol. 14, 31 (2018).

[24] Y. Kang, X. Ni, X. Cheng, A. B. Khanikaev, and A. Z. Genack, Nat. Commun. 9, 3029 (2018).

[25] F. Gao, H. Xue, Z. Yang, K. Lai, Y. Yu, X. Lin, Y. Chong, G. Shvets, and B. Zhang, Nat. Phys. 14, 140 (2018).

[26] T. W. Liu and F. Semperlotti, Phys. Rev. Appl. 11, 014040 (2019).

[27] K. Qian, D. J. Apigo, C. Prodan, Y. Barlas, and E. Prodan, Phys. Rev. B 98, 155138 (2018).

[28] I. Syozi, Prog. Theor. Phys. 6, 306 (1951).

[29] K.-H. Kim, M.-S. Hwang, H.-R. Kim, J.-H. Choi, Y.-S. No, and H.-G. Park, Nat. Commun. 7, 13893 (2016). 
[30] S. G. Johnson and J. D. Joannopoulos, Opt. Express 8, 173 (2001).

[31] M. Saba, J. M. Hamm, J. J. Baumberg, and O. Hess, Phys. Rev. Lett. 119, 227401 (2017).

[32] C. J. Bradley and A. P. Cracknell, The Mathematical Theory of Symmetry in Solids: Representation Theory for Point Groups and Space Groups, revised ed. (Oxford University Press, New York, 2010), p. 745.

[33] M. V. Berry, Proc. R. Soc. London A 392, 45 (1984).

[34] See Supplemental Material at http://link.aps.org/supplemental/ 10.1103/PhysRevResearch.2.012011 for further details on the method used to compute the Berry curvature, the numerical valley Chern number, the transmission spectra, and the chirality map.
[35] A. F. Oskooi, D. Roundy, M. Ibanescu, P. Bermel, J. D. Joannopoulos, and S. G. Johnson, Comput. Phys. Commun. 181, 687 (2010).

[36] P. Lodahl, S. Mahmoodian, S. Stobbe, A. Rauschenbeutel, P. Schneeweiss, J. Volz, H. Pichler, and P. Zoller, Nature (London) 541, 473 (2017).

[37] S. S. Oh, B. Lang, D. M. Beggs, D. L. Huffaker, M. Saba, and O. Hess, in The 13th Pacific Rim Conference on Lasers and Electro-Optics (OSA, 2018), p. Th4H.5.

[38] Lumerical, https://www.lumerical.com/products/fdtd/.

[39] X. Ni, A. Alu, and A. B. Khanikaev, in Active Photonic Platforms IX, edited by G. S. Subramania and S. Foteinopoulou (International Society for Optics and Photonics, SPIE, 2017), Vol. 10345, pp. 95-102. 